\title{
Beneficios de un programa de entrenamiento físico en la síntomatología depresiva y calidad de vida en los pacientes en hemodiálisis
}

\author{
Ester Tomás Bernabeu, Anna Junqué Jiménez, Gorka Iza Pinedo, Inés Luceño Solé, Oscar Paz López, \\ Marisa Lavado Santpere
}

\section{Servicio de Nefrologia. Hospital de Terrassa. Consorci Sanitari Terrassa. Barcelona}

\section{Introducción:}

Los síntomas depresivos son frecuentes en hemodiálisis (HD). Dada la deteriorada calidad de vida de éstos pacientes, la prevención y tratamiento de estos síntomas son de gran importancia. Diversos estudios muestran efectos beneficiosos del ejercicio físico en los síntomas depresivos y la calidad de vida.

\section{Objetivos:}

Analizar el efecto de un programa de ejercicio físico intradiálisis sobre la sintomatología depresiva y calidad de vida en nuestros pacientes en HD.

\section{Material y métodos:}

Estudio prospectivo unicéntrico (12 semanas) con dos grupos comparativos. El grupo ejercicio (ET) incluía un programa de ejercicio físico usando pelotas medicinales, pesas, bandas elásticas y cicloergómetros en las primeras dos horas de HD. Grupo control (C) recibía el cuidado habitual en HD.

Datos analizados:

1. Parámetros bioquímicos.

2. Fuerza extensión máxima quadriceps (FEMQ) y hand-grip (HG).

3. Tests Capacidad funcional: "Sit to stand to sit" (STS10) y "six-minutes walking test" (6MWT).

4. Sintomatología depresiva:Inventario Beck (BDI).

5. Calidad de Vida: EuroQol-5D(EQ-5D).

\section{Resultados:}

23 pacientes incluidos en HD: $56 \%$ hombres. Edad media 71.2 años y 57.6 meses en HD. 9 fueron asignados al ET y 14 al C.No existían diferencias basales significativas entre grupos. Al final del estudio, el grupo ET presentó globalmente una mejoría significativa $\left({ }^{*} p<0,05\right)$ de las pruebas realizadas: $F E M Q *(17.2 \pm 11.9$ vs $21.1 \pm 12.5 \mathrm{~kg})$, $\mathrm{HG} *(25.6 \pm 13.1$ vs $27.3 \pm 12.6 \mathrm{~kg}), \mathrm{STS} 10 *(23 \pm 6.7$ vs $20.3 \pm 5.9 \mathrm{sec}), 6 \mathrm{MWT}^{*}(5 \%, 418.6$ vs $439 \mathrm{~m})$, $\mathrm{BDI}^{*}(11.7 \pm 8.1$ vs $8.4 \pm 5.9)$ y $\mathrm{EQ}-5 \mathrm{D} *(58.7 \pm 13.6$ vs 63.7 \pm 17.7 ). En el grupo $C$, un deterioro significativo se observó para fuerza muscular $(H G * 21.2 \pm 10.1$ vs $19.7 \pm 11.1 \mathrm{~kg}$ ). Test funcionales, BDI y EQ-5D, también empeoraron en grupo $\mathrm{C}$, si bien no se alcanzó significación estadística. No existieron cambios relevantes en la medicación antidepresiva ni en las características de HD a lo largo del estudio.

\section{Conclusiones:}

1. La sintomatología depresiva mejoró tras la realización de programa de ejercicio físico completo intradiálisis en los pacientes en HD de nuestro estudio.

2. Éstos resultados refuerzan los beneficios psicológicos descritos del ejercicio físico en los pacientes en HD.

3. Con los resultados de nuestro estudio, deberíamos considerar la realización de ejercicio físico intradiálisis como una parte más del cuidado integral del paciente renal en HD. 


\section{Referencias Bibliográficas}

1. Blake C, Codd MB, Cassidy A, O'Meara YM. Physical function, employment and quality of life in endstage renal disease. J Nephrol2000;13(2):142-9.

2. Segura-Ortí E, Rodilla-Alama V, Lisón JF. Physiotherapy during hemodialysis: Results of a progressive resistance-training program. Nefrología 2008;28(1):67-72.

3. Goldberg AP, Geltman EM, Hagberg JM, Gavin JR 3rd, Delmez JA, Carney RM, et al. Therapeutic benefits of exercise training for hemodialysis patients. Kidney Int Suppl 1983;16:S303-9.

4. Harter HR, Goldberg AP. Endurance exercise training. An effective therapeutic modality for hemodialysis patients. Med Clin North Am 1985;69(1):159-75.
5. suh MR, Jung HH, Kim SB, Park JS, Yang WS. Effects of regular exercise on anxiety, depression and quality of life in maintenance hemodialysis patients. Ren Fail 2002, 24(3):337-45.

6. Cheema $B$, Singh M. Exercise training in patients receiving maintenance hemodialysis: $A$ systematic review of clinical trials. Am J Nephrol 2005;25(4):352-64.

7. Kouidi E, Iacovides $A$, Iordanis $P$, vassilioiu $S$, Deligiannis A.Exercise renal rehabilitation program: physchosocial effects. Nephron 1997; 77(2):152-8.

8. Craney RM, McKevitt PM, Goldberg AP, Hagberg $J$, Delmez JA, harter HR. Pyschological effects of excercise training in hemodialysis patients. Nephron 1983; 33(3):179-81. 